\title{
Developing an Accurate CFD Based Gust Model for the Truss Braced Wing Aircraft
}

\author{
Robert E. Bartels* \\ NASA Langley Research Center, Hampton, VA, 23681, USA
}

\begin{abstract}
The increased flexibility of long endurance aircraft having high aspect ratio wings necessitates attention to gust response and perhaps the incorporation of gust load alleviation. The design of civil transport aircraft with a strut or truss-braced high aspect ratio wing furthermore requires gust response analysis in the transonic cruise range. This requirement motivates the use of high fidelity nonlinear computational fluid dynamics (CFD) for gust response analysis. This paper presents the development of a CFD based gust model for the truss braced wing aircraft. A sharp-edged gust provides the gust system identification. The result of the system identification is several thousand time steps of instantaneous pressure coefficients over the entire vehicle. This data is filtered and downsampled to provide the snapshot data set from which a reduced order model is developed. A stochastic singular value decomposition algorithm is used to obtain a proper orthogonal decomposition (POD). The POD model is combined with a convolution integral to predict the time varying pressure coefficient distribution due to a novel gust profile. Finally the unsteady surface pressure response of the truss braced wing vehicle to a one-minus-cosine gust, simulated using the reduced order model, is compared with the full CFD.
\end{abstract}

\section{Nomenclature}

Filtered pressure coefficient

Predicted value of pressure coefficient

Unfiltered pressure coefficient

Gust function factor based on aircraft weight ratios

Generalized force due to gust

Gust length, $f t$

Free stream Mach number

Number of structure modes

Number of surface points

Number of snapshots

Number of time steps

Number of proper orthogonal decomposition modes

Free stream dynamic pressure, $p s i$

Integration matrix, $i n^{2}$

Time, sec

Non-dimensional time

Transfer function

Free stream velocity, $f t / s e c$

$z$-direction gust velocity magnitude, $\mathrm{ft} / \mathrm{sec}$

$z$-direction one-minus-cosine gust intensity, $\mathrm{ft} / \mathrm{sec}$

$z$-direction sharp-edged gust intensity, $\mathrm{ft} / \mathrm{sec}$

$z$-direction reference gust intensity, $\mathrm{ft} / \mathrm{sec}$

Wave number

Vector of time coefficients in proper orthogonal decomposition analysis

Predicted value of $\beta$

* Senior Research Engineer, Aeroelasticity Branch, AIAA Senior Member 


$\begin{array}{ll}\delta & \text { Structure mode shape matrix } \\ \Phi & \text { Singular vectors of a proper orthogonal decomposition } \\ \Xi & \text { Vector of training values of dynamic component of surface } \\ \hat{\Xi} & \text { nodal pressure coefficients } \\ & \text { Predicted value of } \Xi\end{array}$

\section{Introduction}

Aerodynamic efficiency increase and drag reduction are key NASA Subsonic Fixed Wing Program goals. ${ }^{1}$ A component of that research program investigates the truss braced wing (TBW) configuration. Multidisciplinary design optimization (MDO) studies of truss braced wing airplanes suggest that optimal designs can have very flexible wings. ${ }^{2}$ It is well known that vehicles with long flexible wings can have aeroelastic issues related to flutter, gust loads, maneuver loads, limit cycle oscillation, ride quality and buckling. ${ }^{3}$ The TBW may also have stability issues with low sweep angles and very low structural frequencies that can couple with aircraft rigid body modes and a flight control system.

Other aircraft development efforts aimed at high endurance have resulted in very flexible wings. Experience in aircraft design programs intended to improve aerodynamic efficiency such as the HiLDA (High Lift-to-Drag Active) wing, ${ }^{4}$ high altitude long endurance (HALE), ${ }^{5}$ and the Aeroenvironment Helios crash investigation ${ }^{6}$ indicate that gust response requires more thorough analysis and validation using nonlinear multidisciplinary aeroservoelastic codes. For these reasons it is likely that many future projects will necessitate a new level of analysis not seen in current production aircraft design practices. Final designs will likely include closed-loop flutter suppression and gust load alleviation. ${ }^{7,8}$

Gust analyses have been a standard part of vehicle loads analysis for many decades. Analysis to date indicates that gust loads and closed-loop gust response of the TBW may be a critical design factor. Production vehicle design practice uses gust analysis with linear aerodynamics. Very sophisticated, but fully linear gust models, have been developed for both time and frequency domain analyses. The Laplace transform of an arbitrary gust wave form has facilitated the development of frequency domain models. Alternately, a reduced order model (ROM) developed using time history data of an appropriate parameter set for an aeroelastic model can be imported into a linearized state-space model for closed-loop analysis. However, the reduced order gust model and the aerodynamic response data needed to construct that model must be generated first. Time domain gust analysis has historically been performed using a panel code in which the introduction of a perturbation velocity as a local angle of attack increment is relatively straight forward. A linear panel code is acceptable if the flow field and unsteady aerodynamic response are entirely linear. Linear gust aerodynamics may or may not be adequate as more flexible vehicles are designed to fly in the transonic flight regime. If the steady state or unsteady flow are nonlinear, a higher order CFD simulation of the gust response will be required.

For this reason there has been an interest in developing gust modeling capability in high fidelity nonlinear CFD codes. A technique called the field velocity method (FVM) has been developed and is widely being implemented for the simulation of a gust within CFD codes. ${ }^{9-12}$ A previous publication has discussed the development of this method in the NASA CFD code FUN3D. ${ }^{13}$ The present paper uses the gust simulation capability in FUN3D to perform gust analysis and develop a gust model for the truss braced wing aircraft. The development of a CFD based gust model also provides an opportunity to explore a new approach to reduced order gust modeling. A method of creating the ROM of a gust is discussed using proper orthogonal decomposition (POD) combined with a convolution of the gust response.

At the same time an accurate gust model based on CFD will provide value added to the truss braced wing (TBW) aircraft design effort. Development of that gust model is the focus of the present paper. The TBW version to be used in this paper is the Subsonic Ultra Green Aircraft Research (SUGAR) High aircraft being developed by the Boeing company. ${ }^{14}$ This vehicle is being designed under a NASA Fixed Wing N+3 NRA contract. This effort is tasked with developing an aircraft that might carry passengers in the next 30 year time frame. By using truss structures the aircraft can be designed with a thinner and lighter inboard wing, thus reducing weight and making possible the use of a higher aspect ratio wing than is used by current civil transports. The increased lift to drag ratio that is anticipated contributes to a reduced fuel usage. The intent in this paper is to develop a reduced order gust model that can be used in a closedloop aeroservoelastic design and analysis of a gust load alleviation control law. The first sections of this paper will briefly discuss the FUN3D code, the CFD model, the field velocity method of modeling gusts, and the approach to creating the reduced order model. The reduced order model results will be compared with a direct FUN3D simulation of a one-minus-cosine gust. 


\section{FUN3D Solver}

The Navier-Stokes code FUN3D (fully unstructured Navier-Stokes three-dimensional) is a finite-volume unstructured CFD code for either compressible or incompressible flows. ${ }^{15,16}$ Flow variables are stored at the vertices of the grid. FUN3D can solve the discrete compressible Euler or Reynolds-averaged Navier-Stokes (RANS) flow equations either tightly or loosely coupled with a turbulence model on mixed element grids, including tetrahedra, prisms, pyramids and hexahedra. The inviscid fluxes are computed using an approximate Riemann solver based on the values on either side of a cell interface. Cell interface values are obtained by a least squares extrapolation using gradients computed at the vertices.

The present study uses the RANS solution capability of FUN3D. The simulations include turbulence provided by loosely coupling the Spalart-Allmaras turbulence model. ${ }^{17}$ In the present study the low dissipation flux splitting scheme for the inviscid flux construction and the Venkatakrishnan limiter are used. The inviscid flux and viscous terms are computed with second order accuracy. The unsteady solutions at each time step are updated with a second order backwards Euler time differencing scheme. Steady state and subiterative solutions are accelerated to convergence by the use of local time stepping. ${ }^{15}$ Domain decomposition is used to enable distributed parallel computing.

\section{The Field Velocity Method and Discrete Gust Model}

The FVM physically introduces gusts into a CFD code by utilizing grid velocity. ${ }^{9-12}$ Normally grid velocity would be associated with physically moving the grid. However, it is possible to introduce an arbitrary perturbation velocity in a stationary grid by prescribing the grid velocity at either all or some of the field grid points without actually moving the grid. For instance, the gust profile can be defined by a streamwise variation in the $z$-direction perturbation velocity such as

$$
w_{g}(x, t)=f_{z}\left(t-x / U_{\infty}\right)
$$

This method has been used for relatively simple to moderately complex configurations, for example Singh and Baeder, ${ }^{11}$ Zaide and Raveh, ${ }^{18}$ Raveh, ${ }^{19,20}$ and Wang et al. ${ }^{21}$ It was recently used to simulate the aeroelastic response of a launch vehicle to a sequence of one-minus-cosine gusts. ${ }^{13}$

In this study a one-minus-cosine and a sharp-edged gust profile will be used. In the present case, having only a $z$-component, the one-minus-cosine gust velocity is

$$
w_{g}(x, t)=\frac{1}{2} w_{\text {omc }}\left[1-\cos \left(\frac{\pi\left(t-x / U_{\infty}\right)}{L_{g} / U_{\infty}}\right)\right] \text { for } t U_{\infty}-2 L_{g}<x<t U_{\infty}
$$

where $L_{g}$ is the gust length and $w_{\text {omc }}$ is the one-minus-cosine gust intensity. In order to obtain the reduced order (ROM) gust model, a time-accurate unsteady solution is computed for the response to a sharp-edged gust. The sharp-edged gust velocity is given by

$$
w_{g}(x, t)=w_{S E G} \text { for } t>x / U_{\infty} \text { and } w_{g}(x, t)=0 \text { for } t<x / U_{\infty} .
$$

\section{Development of a POD/Convolution Based Reduced Order Gust Model}

Rather than computing a fully time-accurate CFD solution for each gust profile, it would be of interest to create a reduced order model that is capable of predicting the time history response of the distributed surface pressures to a gust input. Such a model can be constructed using the convolution of a sharp-edged gust, combined with a model constructed from the proper orthogonal decomposition of the covariance matrix of the sharp-edged gust unsteady pressure coefficients. In this model, the POD eigenvectors provide the spatial distribution of the principle components of the unsteady pressure response. Combining the POD eigenvectors with a convolution integral provide a method of predicting the unsteady surface pressure responses to an arbitrary gust.

A sharp-edged gust (SEG) velocity profile is used to generate the pressure response training data. For an array of surface forces that are continuous in time, the aeroelastic generalized forces due to a gust can be written as

$$
G_{S E G}(t)=[\delta]^{T} \hat{f}(t)
$$

where $\hat{f}$ are the aerodynamic nodal forces derived from the pressures at CFD surface grid points and $[\delta]$ are the structural mode shapes. The time history of generalized forces due to an arbitrary gust input can be constructed by 
convolution, namely

$$
G_{g}(t)=\int_{0}^{t} \dot{w}_{g}(\tau) G_{S E G}(t-\tau) d \tau
$$

As Raveh does, it is possible to compute the generalized force for any arbitrary gust using the generalized force $G_{S E G}$ due to the sharp-edged gust. ${ }^{20}$ On the other hand, it is useful to compute the convolution using a POD model of the unsteady pressures. By using a POD, the nodal pressure coefficients due to the sharp-edged gust can be decomposed into the spatial component of the POD $\Phi$ and the time varying component $\beta(t)$. Due to an arbitrary gust, the nodal forces are

$$
\hat{f}(t)=q_{\infty}[s][\Phi] \hat{\beta}(t)
$$

(the hat indicates the predicted value) and the generalized forces are

$$
G_{g}(t)=[P] \int_{0}^{t} \dot{w}_{g}(\tau) \beta_{S E G}(t-\tau) d \tau=[P] \hat{\beta}(t)
$$

where $[P]=q_{\infty}[\delta]^{T}[s][\Phi]$.

The discrete time formulation of the $\mathrm{POD} /$ convolution requires additional discussion. A proper orthogonal decomposition of the surface pressures for all time steps of an entire CFD simulation is out of the realm of possibility except for a very coarse mesh on a simple geometry. The present aircraft mesh has nearly 500,000 surface nodes, which by today's standards is not an excessively refined grid. At the present refinement, it has been found necessary to use a subset, or snapshots of the surface pressure time history. The POD eigenvectors are computed by using the matrix of pressure coefficient data at $N_{s s}$ snapshots

$$
[X]^{T}=\left[\begin{array}{lllll}
\Xi_{1} & \cdots & \Xi_{l} & \cdots & \Xi_{N_{s s}}
\end{array}\right] .
$$

The columns of this matrix are composed of the surface node pressure coefficients at a given snapshot. The snapshot $l$ of the training data is

$$
\Xi_{l}=\left[\begin{array}{c}
c_{p_{1, l}} \\
\vdots \\
c_{p_{N_{s}, l}}
\end{array}\right] .
$$

The relationship between the array of pressure coefficients and the POD model at time step $l$ is given by $\Xi_{l}=[\Phi] \beta_{l}$. The matrix $\Phi$ is the collection of singular vectors of the matrix $[X]^{T}$. The size of the matrix $\Phi$ is reduced to form a truncated POD-basis $\Phi_{r}$ with dimension $N_{s} \times N_{P O D}$ by retaining only the eigenvectors associated with the largest eigenvalues. Although it is possible to find the eigenvectors of the covariance matrix, in practice, especially for large systems, computing the eigenvectors from a singular value decomposition (SVD) is more efficient and robust. The rank-r approximation of the matrix $X^{T}$ is obtained as an approximate solution to

$$
X^{T} \approx \Psi \Sigma \Phi_{r}^{T}
$$

by an SVD. The orthonormal vectors $\psi_{1}, \psi_{2}, \ldots, \psi_{r}$ are the approximate left singular vectors and the orthonormal vectors $\phi_{1}, \phi_{2}, \ldots, \phi_{r}$ are the approximate right singular vectors, while $\sigma_{1} \geq \sigma_{2} \geq, \ldots, \sigma_{r}$ are the singular values of $X^{T}$. The matrix $\Phi_{r}$ contains the leading $r$ right singular vectors of the matrix $X^{T}$. A very efficient stochastic algorithm for computing the singular value decomposition of large systems has been developed in Liberty et al. ${ }^{22}$ and Rokhlin et al. ${ }^{23}$ This algorithm will be used and is discussed further in the next section. Finally, if the POD modes are unit normalized so that $\Phi^{T} \Phi=I$, the time varying component of the POD model of the training data can be obtained for time step $l$ by $\beta_{S E G, l}=\left[\Phi_{r}\right]^{T} \Xi_{l}$.

The $N_{s s}$ snapshots of training data are a subset constructed by taking CFD surface pressure coefficients at constant intervals over the entire sharp-edged gust simulation. The downsampling of a data set to obtain snapshots requires an anti-aliasing filter so as not to corrupt the data and potentially destablize a numerical scheme with unwanted higher frequencies. Filtering does not appear to be the standard practice in the literature when creating POD models from CFD pressure data. It would seem to be something of growing importance, however, as more CFD models include detached eddy simulation (DES) or large eddy simulation (LES) turbulence models and as larger grid sizes require data downsampling. The filtering used here is by Lele. ${ }^{24}$ The filtered surface pressure data is computed using the sixth-order scheme

$$
\begin{gathered}
b c_{p, i-2}+a c_{p, i-1}+c_{p, i}+a c_{p, i+1}+b c_{p, i+2}=A f_{i}+\frac{D}{2}\left(\tilde{c}_{p, i+3}+\tilde{c}_{p, i-3}\right)+\frac{C}{2}\left(\tilde{c}_{p, i+2}+\tilde{c}_{p, i-2}\right)+\frac{B}{2}\left(\tilde{c}_{p, i+1}+\tilde{c}_{p, i-1}\right) \\
\stackrel{4 \text { of } 15}{(15}
\end{gathered}
$$


The $\tilde{c}_{p, i}$ are the unfiltered pressure coefficients while the $c_{p, i}$ are the filtered values for $i: 1 \rightarrow N_{s s}$. The coefficient $a=0.4999$ was chosen to give the best behavior and $b=(3-2 a) / 10, A=(2+3 a) / 4, B=(6+7 a) / 8, C=(6+a) / 20$, $D=(2-3 a) / 40$.

The convolution is integrated with a discrete method of the form

$$
\hat{\beta}_{l}=\sum_{k=1}^{l-1}\left\{c_{k-1} \dot{w}_{g, k-1} \beta_{S E G, l-k+1}+c_{k} \dot{w}_{g, k} \beta_{S E G, l-k}+c_{k+1} \dot{w}_{g, k+1} \beta_{S E G, l-k-1}\right\} \Delta \tau
$$

where the coefficients $c_{k}$ are chosen to give second order accuracy. Surface pressures can be reconstructed for an arbitrary gust by

$$
\hat{\Xi}_{l}=\left[\Phi_{r}\right] \hat{\beta}_{l}
$$

\section{Stochastic Algorithm for Computing Singular Value Decomposition}

As the size of CFD models becomes larger, there is a growing need to implement memory and computing efficient algorithms for performing the proper orthogonal decomposition. There are several methods available to perform the factorization in equation 10, however, a very efficient and robust method is the stochastic algorithm of Liberty et al. ${ }^{22}$ and Rokhlin et al. ${ }^{23}$ and Halko et al. ${ }^{25}$ The source code developed by those authors, and available from the home page of one of the authors, is used in the present computations. The brief description of the algorithm is given here that follows Martinsson. ${ }^{26}$ Let $A=X^{T}$. Sample matrices can be computed

$$
Y=A \Omega \quad \text { and } \quad Z=A^{T} \Theta
$$

where $\Omega$ and $\Theta$ are random matrices. Orthonormal matrices $Q$ and $W$ can be found so that

$$
Y=Q Q^{T} Y \quad \text { and } \quad Z=W W^{T} Z
$$

Solve the linear systems

$$
Q^{T} Y=T\left(W^{T} \Omega\right) \quad \text { and } \quad W^{T} Z=T^{T}\left(Q^{T} \Theta\right)
$$

for $T$. Factor $T$ such that

$$
T=\hat{U} \Sigma \hat{V}^{T}
$$

The desired orthonormal matrices are found by setting

$$
U=Q \hat{U} \quad \text { and } \quad V=W \hat{V} \quad .
$$

The matrix $V$ contains the desired eigenvectors. This method has been successfully used for extremely large systems. Previous studies by the authors have shown it to be very efficient from a memory and CPU usage standpoint compared to standard methods of singular value decomposition.

\section{Results}

The TBW outer mold line used in the present analysis is shown in Figure 1. The CFD volume mesh was generated using VGRID 4.00. ${ }^{27}$ It is a half-span tetrahedral mesh with 17.3 million nodes with mirror boundary conditions at the vehicle symmetry plane. The surface is modeled with 478,000 nodes. The normal spacing at the wall is approximately at $y+=1$ everywhere. Figure 2 shows the surface mesh clustering in the wing and engine nacelle regions. This paper will investigate the development of a gust model that can be used in a future closed-loop aeroervoelastic analysis of the truss braced wing aircraft. The focus of the present analysis is a flight condition at which Boeing analyses have resulted in the largest gust induced wing bending moment. That gust is a one-minus-cosine gust with gust length of $110 \mathrm{ft}$ and gust intensity of $31.4 \mathrm{ft} / \mathrm{sec}$. The Boeing aeroelastic analysis was done with MSC.Nastran ${ }^{T M}$ using doublet lattice aerodynamics and a linear structural model. The condition at which that analysis was done is Mach 0.70 at an altitude of $19 \mathrm{k} \mathrm{ft}$ and zero degrees angle of attack. The present rigid vehicle gust analysis uses the same flight condition.

The gust simulations are initialized with a steady state solution. Steady state solutions were obtained after 30,000 iterations using Courant-Friedrichs-Lewy (CFL) number of 10 for the flow and 6 for the turbulence equations. The 
steady state lift coefficient varied less than $10^{-5}$ over the last 5000 iterations. Likewise, the moment coefficient varied less than $10^{-4}$ over the last 5000 iterations. Both the lift and moment coefficients had jitter that continued to the end, but that was also extremely small in comparison to absolute values of the coefficients. This jitter had been attenuated somewhat by the use of the Venkatakrishnan limiter but was still present. That jitter also shows up minutely in the unsteady pressure coefficient time histories. Returning to the steady state solution, the surface pressure distribution on the aircraft is shown in Figure 3(a). Note that the model includes engine nacelle with intake and both fan and core exhaust planes. Inlet and exhaust conditions are set in the present computations to produce reasonably realistic flow conditions around the engine. Contours of streamwise velocity around the engine are shown in Figure 3(b). The exhaust flow from the engine core is evident from the colored contours in that figure.

For the unsteady simulations a dimensional time step of $3.2 \times 10^{-4} \mathrm{sec}$ was used. Twenty sub-iterations per time step with a CFL number of 15 for the flow and 8 for the turbulence model were used. Pressure coefficient data was written at each time step. The complete time history of data included 3000 time steps. This many data samples with a surface of nearly 500,000 nodes proved to be too large a data set to reasonably compute the SVD on a single computing node. This computing limitation required that the pressure coefficient data be downsampled to something less than 500 snapshots. For this reason, the surface pressure data was repeatedly filtered and downsampled by two with the method described earlier, to a subset of every eighth point in time. This left a data sample with 376 snapshots. An alternate downsampled data set was also extracted that was unfiltered. The POD analysis was next performed on the two downsampled data sets using the stochastic SVD software by Liberty et al. ${ }^{22}$ and Rokhlin et al. ${ }^{23}$ Models with 10, 20, 30 and 40 POD modes were developed. It should be noted that a standard method of SVD was also used with which to compare. The stochastic algorithm easily out performed the standard SVD algorithm in execution time. The solutions by the two methods were nearly identical.

A global error analysis was performed by comparing the predicted surface pressure time histories with the original surface pressure time histories due to the sharp-edged gust. A plot of the global error from the filtered and unfiltered data sets as a function of the number of POD modes is shown in Figure 4. It can be noted that the addition of modes significantly reduces the error, at least in the case of the filtered data. The unfiltered data error reduces initially, but clearly the return diminishes as the number of modes increases.

A comparison of the time histories of the ROM predicted and FUN3D simulated surface pressures at a collection of points will be shown for the one-minus-cosine gust. Figure 5 shows the point stations at which time histories will be presented. Several stations are chosen on the upper wing surface, several on the upper surface of the strut, one on the engine nacelle and one on the horizontal tail. Figures 6(a)-(f) show surface pressure coefficient time histories. The values plotted are $c_{p}^{\prime}=\left(c_{p}-c_{p_{s s}}\right) / c_{p_{0}}$ where $c_{p_{s s}}$ is the steady state value. The parameter $c_{p_{0}}$ is a normalization factor that is used consistently throughout the time histories. Time histories are shown for the FUN3D simulation and for the 40 POD mode convolution responding to the one-minus-cosine gust. Station 1 and 6 are for the wing upper surface. The $c_{p}^{\prime}$ time histories at those stations indicate a strong negative pressure dip as the gust passes. Station 2 is on the horizontal tail. The time delay of the pressure response at the tail is evident by comparing Figure 6(b) with (a) or (f). The strut responses are found in Figure 6(c) and (d). As expected the flow field does not respond as strongly in the strut region as it does over the wing. However, strut station 4 in Figure 6(d) clearly is seeing the influence of the under side of the wing that induces a positive pressure response. As can be seen in the figure, the FUN3D and the predicted time histories compare very well for all the stations shown. Time histories of the lift and moment coefficients given by the 40 mode ROM model and the FUN3D simulation for the one-minus-cosine gust are shown in Figures 7- 8 . The values plotted are $C_{\xi}^{\prime}=\left(C_{\xi}-C_{\xi_{s s}}\right) / C_{\xi_{0}}$ where $C_{\xi_{s s}}$ is the steady state value, $C_{\xi_{0}}$ is a normalization factor and $\xi=(L, M)$ for lift and moment coefficient. The lift and moment coefficients are computed using equation 7 . The perturbation due to the gust is modeled very well by the present ROM model. Both the pressure coefficient and the lift and moment coefficient time histories show what appears to be drift in the FUN3D solution, as seen toward the end of the time trace. Since the FUN3D solution is fully nonlinear, it may be that there is some drift in the solution not evident in the linear ROM model.

It is of interest to know to what degree the gust simulated here is attenuated by the filtering of equation 11 . To investigate the behavior of the filter, the transfer function of equation 11 can be written, following Lele ${ }^{24}$

$$
T(w)=\frac{A+B \cos (w)+C \cos (2 w)+D \cos (3 w)}{1+2 a \cos (w)+2 b \cos (2 w)} \quad \text { where } \quad w=2 \pi k / N
$$

and where $w$ is the wave number. The transfer function of the filter (equation 11) for the values used here is shown in Figure 9 for the range of wave numbers up to the Nyquist frequency. The wave number based on the one-minus-cosine gust length of $110 \mathrm{ft}$ is $w \approx 0.053$ for the present data set. The gust response fundamental frequency falls well within

$$
6 \text { of } 15
$$


the range $(0<w<1.5)$ in which signals are returned essentially unattenuated. It can also be seen from the figure that at the maximum wave number and above, signals are strongly attenuated, as desired. For reassurance one can compare the pressure coefficient time histories for the filtered and unfiltered ROM simulated gust. Figure 10 presents a comparison of the filtered and unfiltered pressure coefficient time histories at station 6 as reconstructed by the present ROM. The filtered and unfiltered responses are nearly identical. In the future, it may be possible to find an anti-aliasing filter that is better fitted in general for that purpose, but for the present one-minus-cosine gust, the current filter seems to be adequate.

A Federal Aviation Regulation ( FAR) family of gusts required for certification has the form

$$
w_{\text {omc }}=w_{r e f} F_{g}\left(\frac{L_{g}}{350}\right)^{1 / 6}
$$

where $w_{r e f}$ is specified based on altitude and $F_{g}$ is a factor based on aircraft weight ratios. Having a reduced order gust model enables an analysis of the effect of parametric variations of gust parameters on the vehicle response. The variation of gust response with gust length $L_{g}$ has been calculated for the truss brace wing aircraft at Mach 0.70 and $19 k f t$. A family of lift and moment coefficient responses are shown in Figures 11 (a) and (b). The lines for $L_{g}=110$ $f t$ correspond to the gust giving maximum wing bending moment for the flexible vehicle according to current linear analyses. The lift and moment coefficient have been normalized to the response for this gust length. For the rigid aircraft, longer gust lengths result in larger vehicle lift and moment responses but also longer time over which loading builds. Of course, to get the actual vehicle response and importantly wing bending moment, the effect of vehicle flexibility will be required as well.

\section{Conclusions}

A computational fluid dynamics based gust model has been developed for the Boeing truss braced wing aircraft. A sharp-edged gust and a one-minus-cosine gust have been simulated using the computational fluid dynamics code FUN3D. The unsteady pressures from the computational fluid dynamics sharp-edged gust simulation are used to create a reduced order model from which the surface pressures of any arbitrary gust can be reproduced. A proper orthogonal decomposition enables a reduction in the size of the reduced order model. A convolution of the sharp-edged gust response enables a prediction of the response to an arbitrary gust. The combined proper orthogonal decomposition and convolution reduced order model enables the prediction of the distributed surface pressure time history due to an arbitrary gust. As the size of computational fluid dynamics models increases, algorithmic efficiency will become increasingly important. A stochastic singular value decomposition algorithm is used to calculate the principle components of the aerodynamic response. This algorithm is found to perform well for the large data set used here. Even with the current moderate number of surface nodes, downsampling of the unsteady pressure data was required. A digital anti-aliasing filter was used in the filter/downsample step. For the present one-minus-cosine gust, the filtered and unfiltered pressure responses were found to be nearly identical. As computational fluid dynamics models increase in size (requiring additional downsampling) or as detached eddy or large eddy simulation turbulence models are used, it is anticipated that filtering will become an important topic. The reduced order and the FUN3D modeled surface pressures and generalized force response to the one-minus-cosine gust are found to agree well. The reduced order gust model is used to compute a family of one-minus-cosine gust responses for the rigid truss braced wing aircraft. It is anticipated that the surface pressure responses from these simulations will be used to construct a closed-loop reduced order model of the flexible truss braced wing aircraft. 


\section{References}

1“National Science and Technology Council: National Aeronautics Research and Development Plan,” Committee report, Feb. 2010.

${ }^{2}$ Gur, O., Bhatia, M., Schetz, J., Mason, W., Kapania, R., and Mavris, D., "Design Optimization of a Truss-Braced-Wing Transonic Transport Aircraft," Journal of Aircraft, Vol. 47, No. 6, 2010, pp. 1907-1917.

${ }^{3}$ Bhatia, M., Gur, O., Kapania, R., Mason, W., Schetz, J., and Haftka, R., "Progress Towards Multidisciplinary Design Optimization of Truss Braced Wing Aircraft with Flutter Constraints," 13th AIAA/ISSMO Multidisciplinary Analysis Optimization Conference, AIAA-2010-9077.

${ }^{4}$ Silva, W. A., Vartio, E., Shimko, A., Kvaternik, R. G., Eure, K. W., and Scott, R. C., "Development of Aeroservoelastic Analytical Models and Gust Load Alleviation Control Laws of a SensorCraft Wind-Tunnel Model Using Measured Data," 47th AIAA/ASME/ASCE/AHS/ASC Structures, Structural Dynamics and Materials Conference, AIAA-2006-1935.

${ }^{5}$ Scott, R., Castelluccio, M., Coulson, D., and Heeg, J., "Aeroservoelastic Wind-Tunnel Tests of a Free-Flying, Joined-Wing SensorCraft Model for Gust Load Alleviation," 52nd AIAA/ASME/ASCE/AHS/ASC Structures, Structural Dynamics and Materials Conference, AIAA-20111960.

${ }^{6}$ Noll, T. E., Brown, J. M., Perez-Davis, M. E., Ishmael, S. D., Tiffany, G. C., and Gaier, M., "Investigation of the Helios Prototype Aircraft Mishap. Volume 1,” Mishap report, NASA, Jan. 2008.

${ }^{7}$ Patil, M. J. and Taylor, D. J., "Gust Response of Highly Flexible Aircraft," 47th AIAA/ASME/ASCE/AHS/ASC Structures, Structural Dynamics, and Materials Conference, AIAA-2006-1638.

${ }^{8}$ Shearer, C. M. and Cesnik, C. E. S., "Nonlinear Flight Dynamics of Very Flexible Aircraft," Journal of Aircraft, Vol. 44, No. 5, 2007, pp. $1528-1545$.

${ }^{9}$ Raveh, D. E., "CFD-Based Models of Aerodynamic Gust Response," 47th AIAA/ASME/ASCE/AHS/ASC Structures, Structural Dynamics, and Materials Conference, AIAA 2006-2022.

${ }^{10}$ Zaide, A. and Raveh, D. E., "Numerical Simulation and Reduced-Order Models for Gust-Response Analysis of Flexible Wings," 17th AIAA Fluid Dynamics Conference, AIAA 2005-5128.

${ }^{11}$ Singh, R. and Baeder, J. D., "Direct Calculation of Three-Dimensional Indicial Lift Response Using Computational Fluid Dynamics," Journal of Aircraft, Vol. 34, No. 4, 1997, pp. 465-471.

${ }^{12}$ Parameswaran, V. and Baeder, J. D., "Indicial Aerodynamics in Compressible Flow-Direct Computational Fluid Dynamic Calculations," Journal of Aircraft, Vol. 34, No. 1, 1997, pp. 131-133.

${ }^{13}$ Bartels, R. E., "Development, Verification and Use of Gust Modeling in the NASA Computational Fluid Dynamics Code FUN3D," NASA TM 217771, October 2012.

${ }^{14}$ Bradley, M., "Subsonic Ultra Green Aircraft Research (SUGAR)," NASA Subsonic Fixed Wing Proposal 08-SSFW1-10-2-0007, The Boeing Company, 2010.

${ }^{15}$ Anderson, W. K. and Bonhaus, D. L., "An Implicit Upwind Algorithm for Computing Turbulent Flows on Unstructured Grids," Computers and Fluids, Vol. 23, No. 1, 1994, pp. 1-22.

${ }^{16}$ NASA LaRC, Hampton, VA, FUN3D Manual, July 2012, http: / fun3d. larc. nasa.gov.

${ }^{17}$ Spalart, P. R. and Allmaras, S. R., "A One-Equation Turbulence Model for Aerodynamic Flows," 30th AIAA Aerospace Sciences Meeting and Exhibit, AIAA 1992-0439.

${ }^{18}$ Zaide, A. and Raveh, D. E., "Numerical Simulation and Reduced-Order Modeling of Airfoil Gust Response," AIAA Journal, Vol. 44, No. 8 , 2006, pp. 1826-1834.

${ }^{19}$ Raveh, D. E., "Gust Response Analysis of Free Elastic Aircraft in the Transonic Flight Regime," Journal of Aircraft, Vol. 48, No. 4, 2011, pp. 1204-1211.

${ }^{20}$ Raveh, D. E., "CFD-Based Gust Response Analysis of Free Elastic Aircraft,” ASD Journal, Vol. 2, No. 1, 2010, pp. $23-34$.

${ }^{21}$ Wang, Z., Zhang, Z., Chen, P. C., and Sarhaddi, D., "A Compact CFD-Based Reduced Order Modeling for Gust Analysis," 52th AIAA/ASME/ASCE/ASC Structures, Structural Dynamics and Materials Conference, AIAA-2011-2041.

${ }^{22}$ Liberty, E., Woolfe, F., Martinsson, P.-G., Rokhlin, V., and Tygert, M., "Randomized algorithms for the low-rank approximation of matrices," Proceedings of the National Academy of Science, Vol. 104, 2007, pp. 20167-20172.

${ }^{23}$ Rokhlin, V., Szlam, A., and Tygert, M., "A randomized algorithm for principal component analysis," Cornell University Library, arXiv.org 0809.2274, 2008.

${ }^{24}$ Lele, S. K., "Compact Finite Difference Schemes with Spectral-like Resolution,” Journal of Computational Physics, Vol. 103, 1992, pp. 1642.

${ }^{25}$ Halko, N., Martinsson, P. G., and Tropp, J., "Finding structure with randomness: Stochastic algorithms for constructing approximate matrix decompositions," Cornell University Library, arXiv.org 0909.4061, 2009.

${ }^{26}$ Martinsson, G., "Randomized Methods for Computing the Singular Value Decomposition (SVD) of Very Large Matrices," Presentation, 2010.

${ }^{27}$ Pirzadeh, S. Z., "Advanced Unstructured Grid Generation for Complex Aerodynamic Applications," 26th AIAA Applied Aerodynamics Conference, AIAA 2008-7178. 


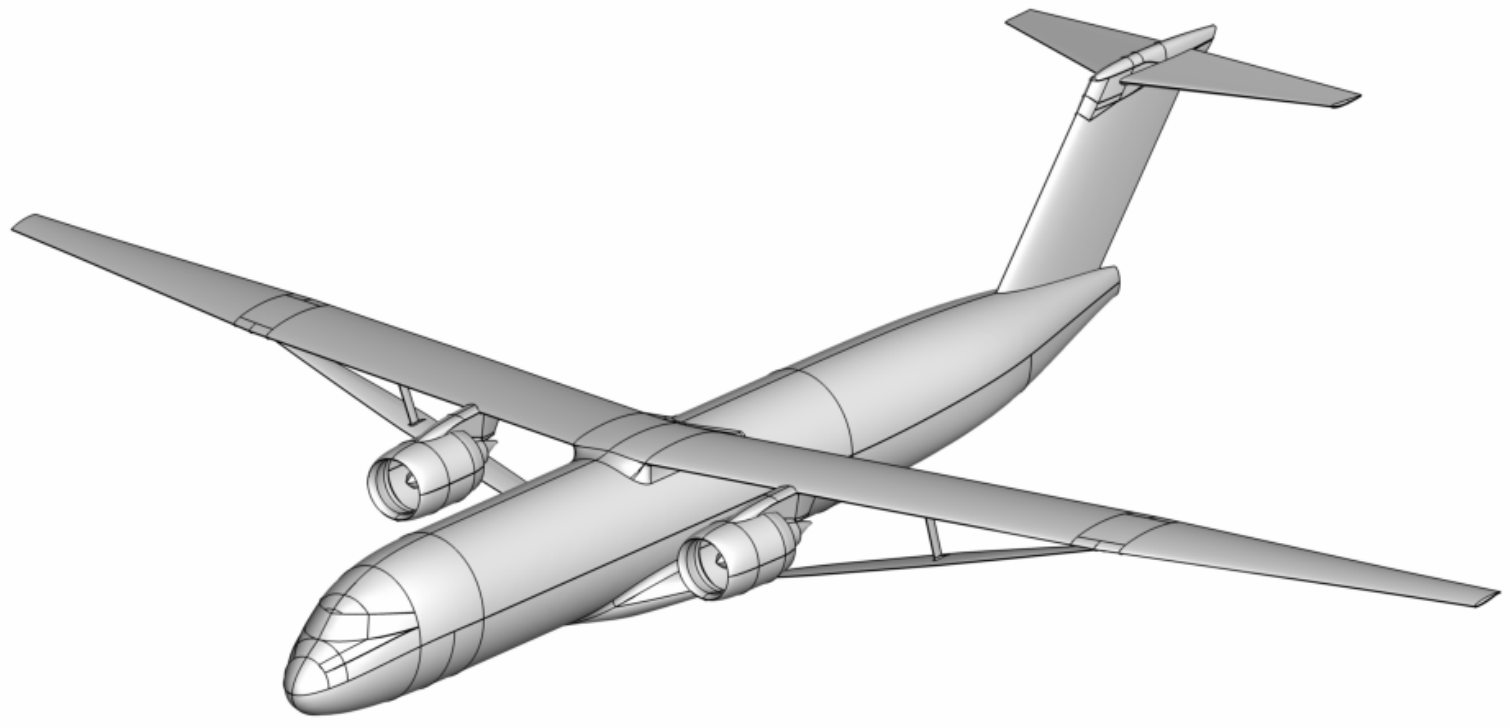

Figure 1. Boeing SUGAR High truss braced wing aircraft. 


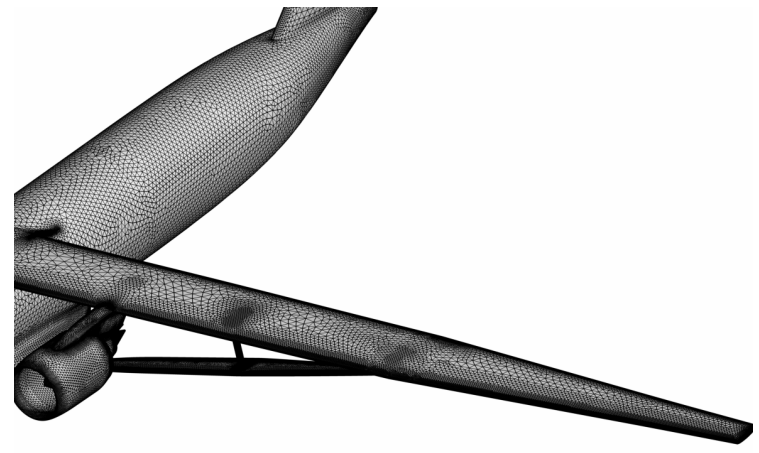

(a) Wing surface mesh

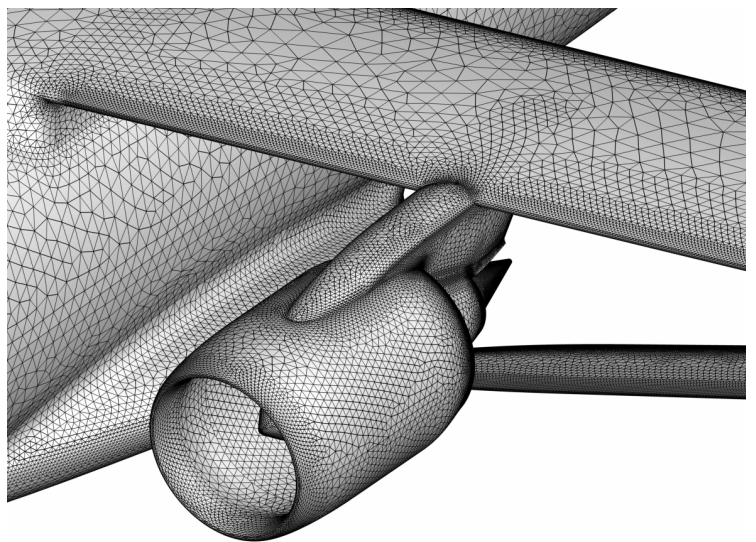

(b) Nacelle surface mesh

Figure 2. Surface mesh.

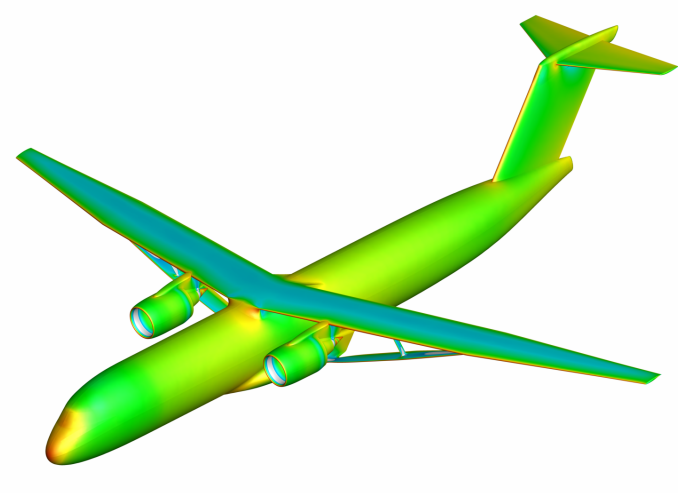

(a) Surface pressure coefficient contours

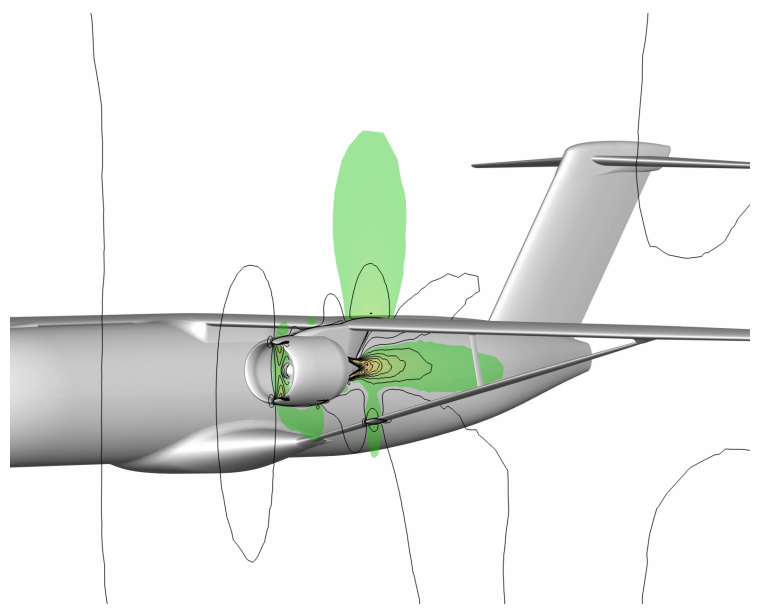

(b) Streamwise velocity contours

Figure 3. Steady state contours, Mach 0.70, $\alpha=0$ deg. 


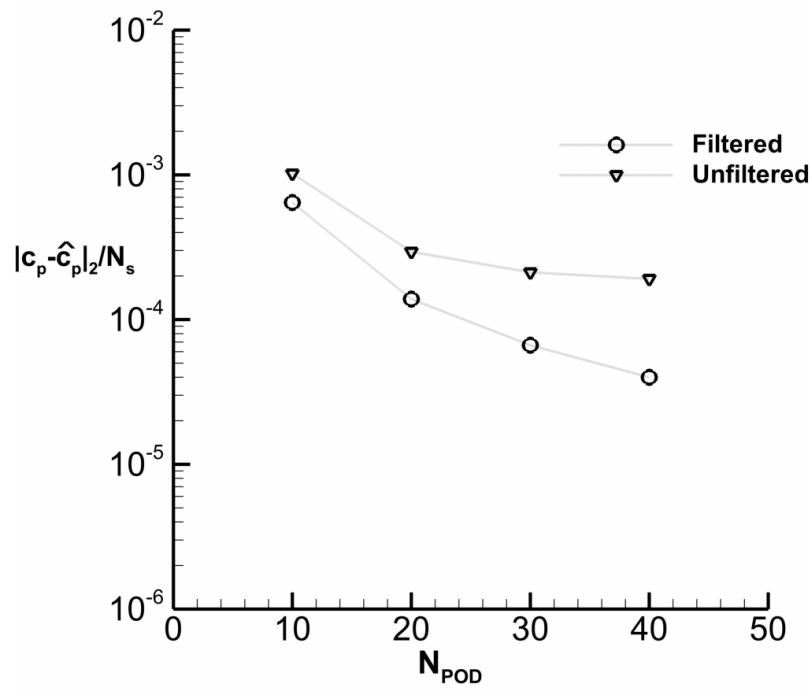

Figure 4. Global error of proper orthogonal decomposition of the sharp-edged gust.

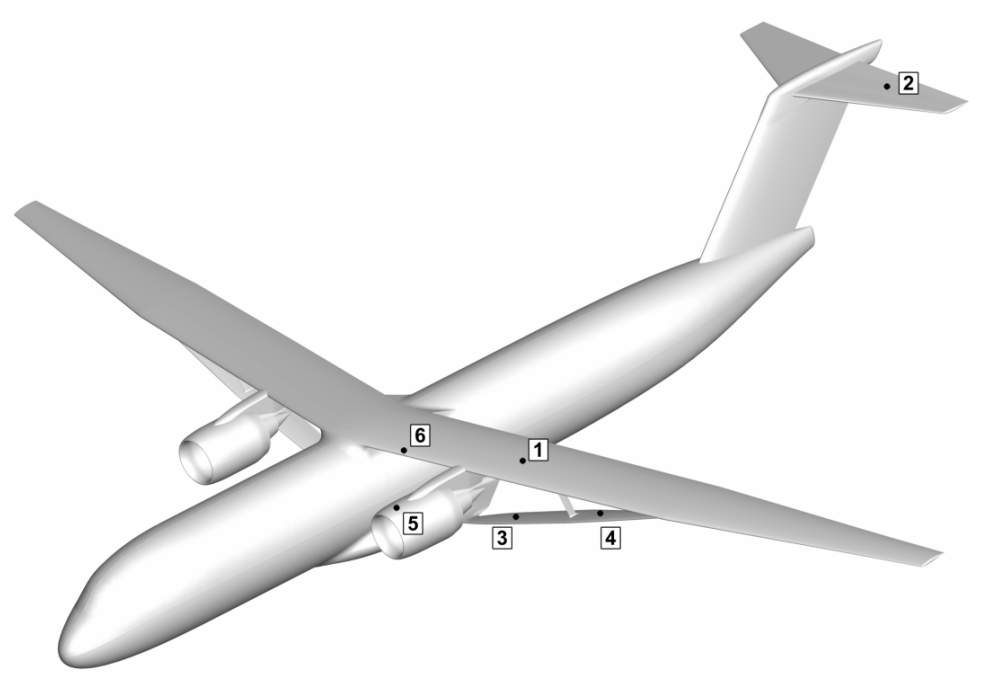

Figure 5. Stations at which $c_{p}$ time histories are plotted. 


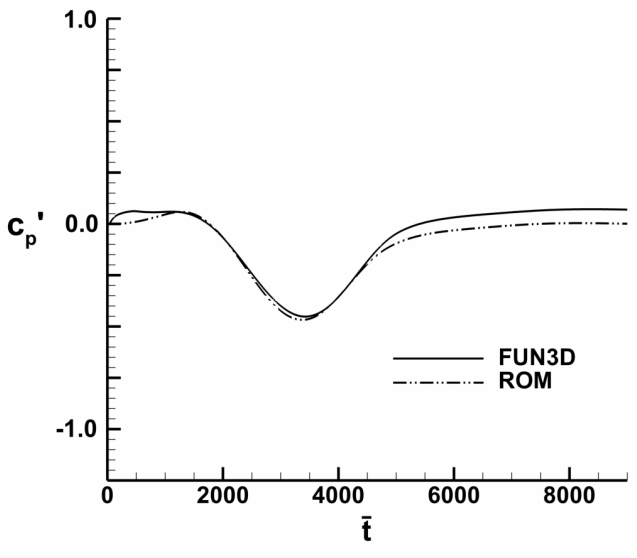

(a) Station 1, wing upper surface

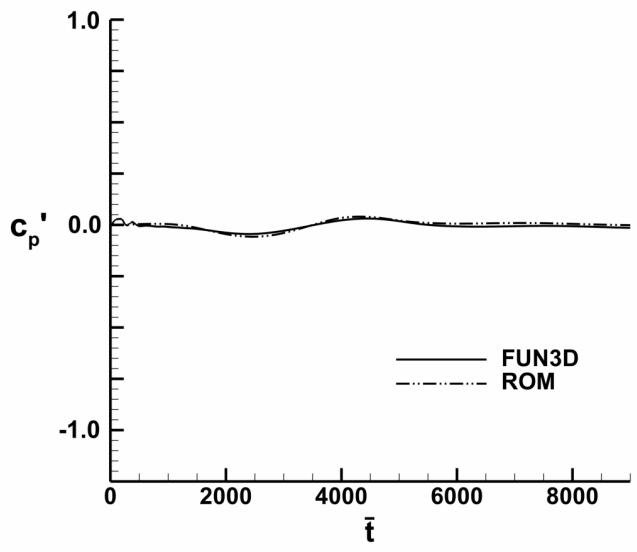

(c) Station 3, wing strut

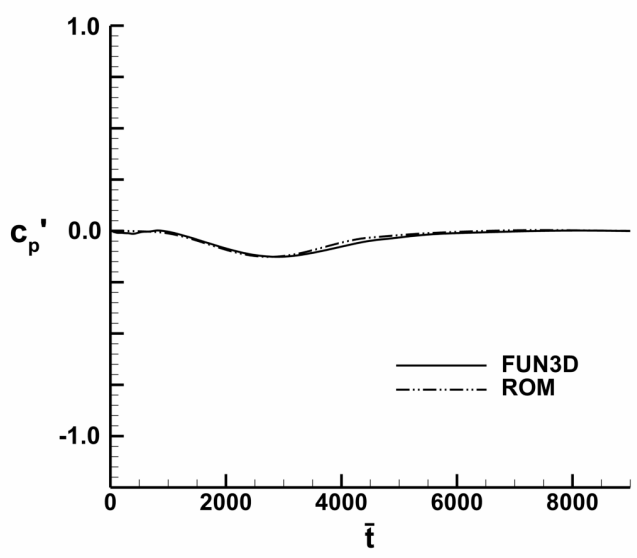

(e) Station 5, engine nacelle

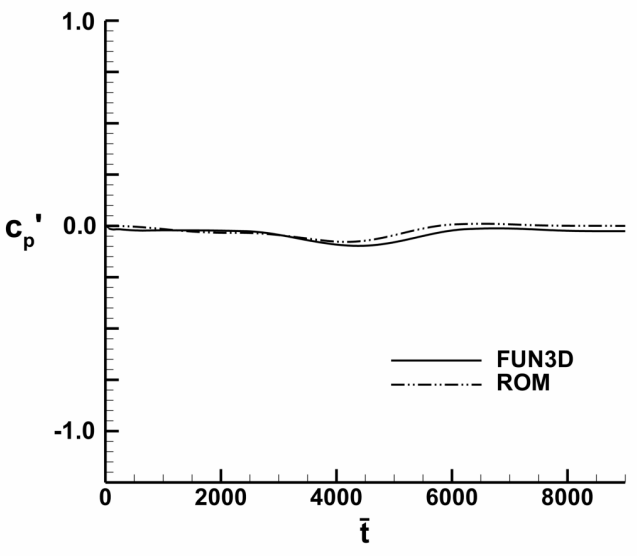

(b) Station 2, horizontal tail

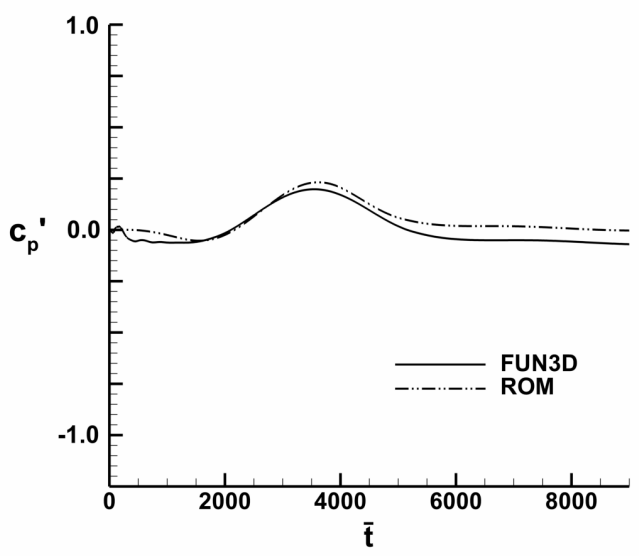

(d) Station 4, wing strut

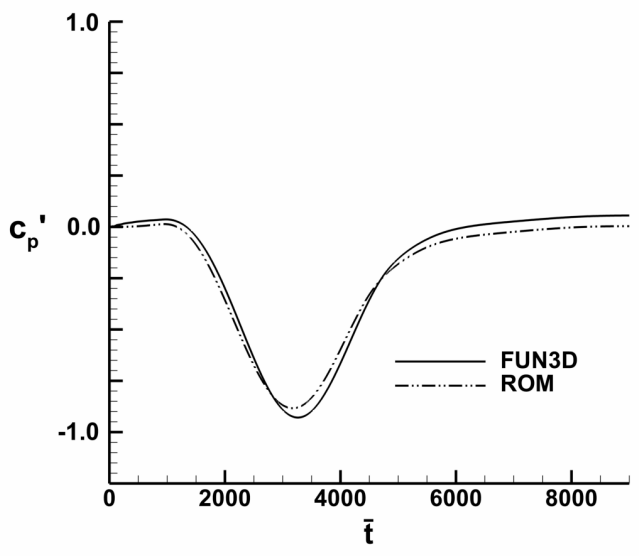

(f) Station 6, wing upper surface

Figure 6. Pressure coefficient time histories due to one-minus-cosine gust (ROM uses equation 13 with 40 modes derived from filtered data).

$$
12 \text { of } 15
$$




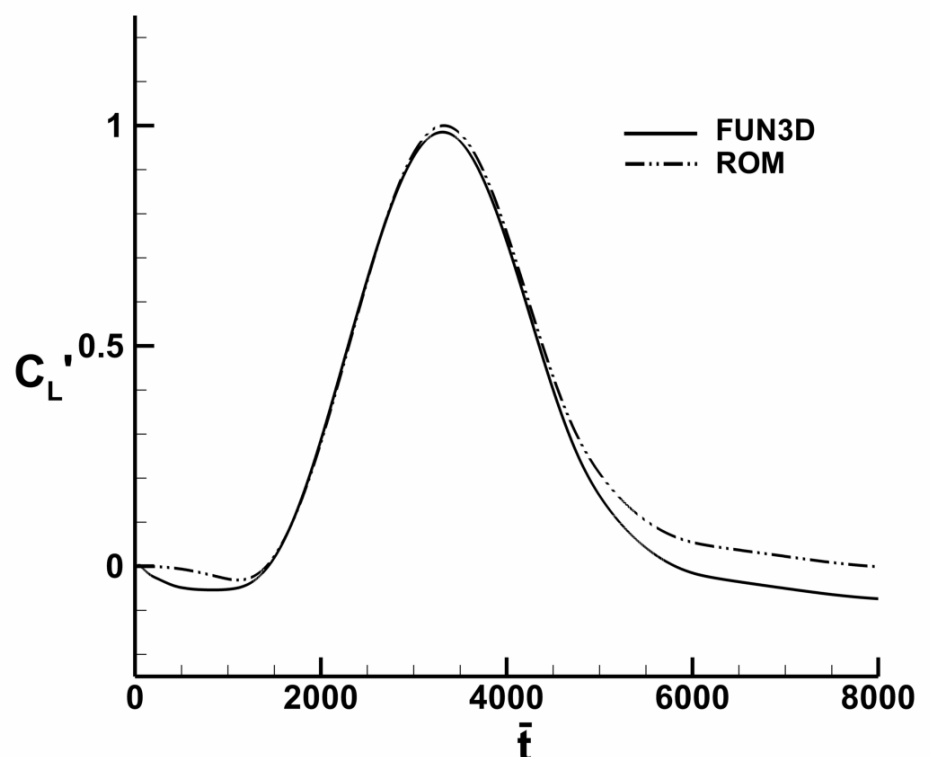

Figure 7. Lift coefficient time history due to one-minus-cosine gust (ROM uses equation 7 with 40 modes derived from filtered data).

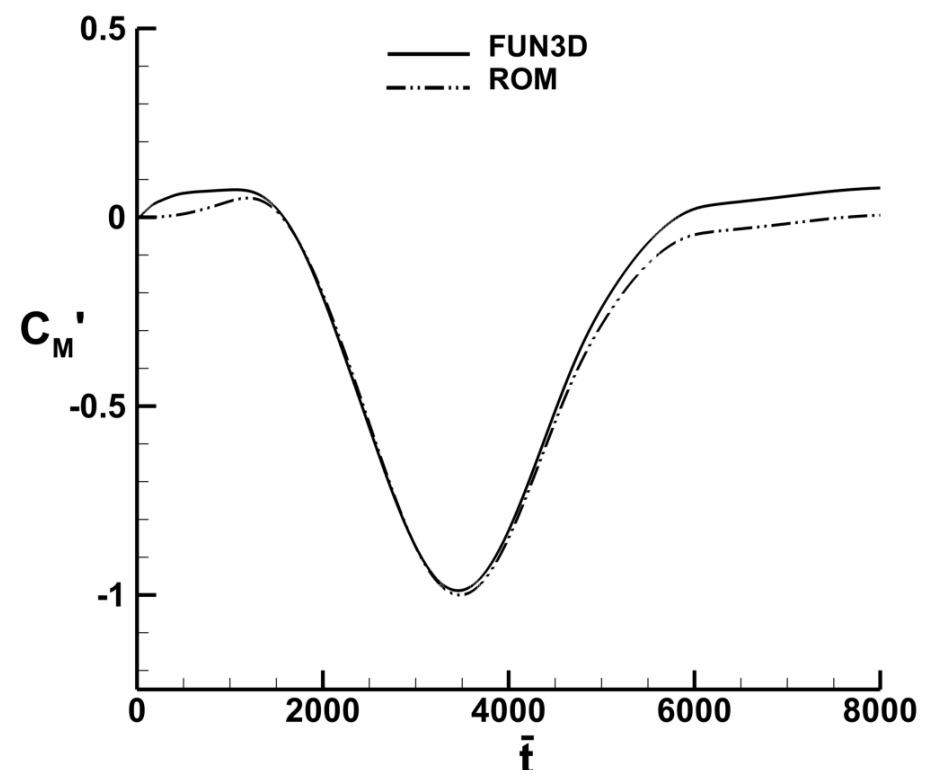

Figure 8. Moment coefficient time history due to one-minus-cosine gust (ROM uses equation 7 with 40 modes derived from filtered data). 


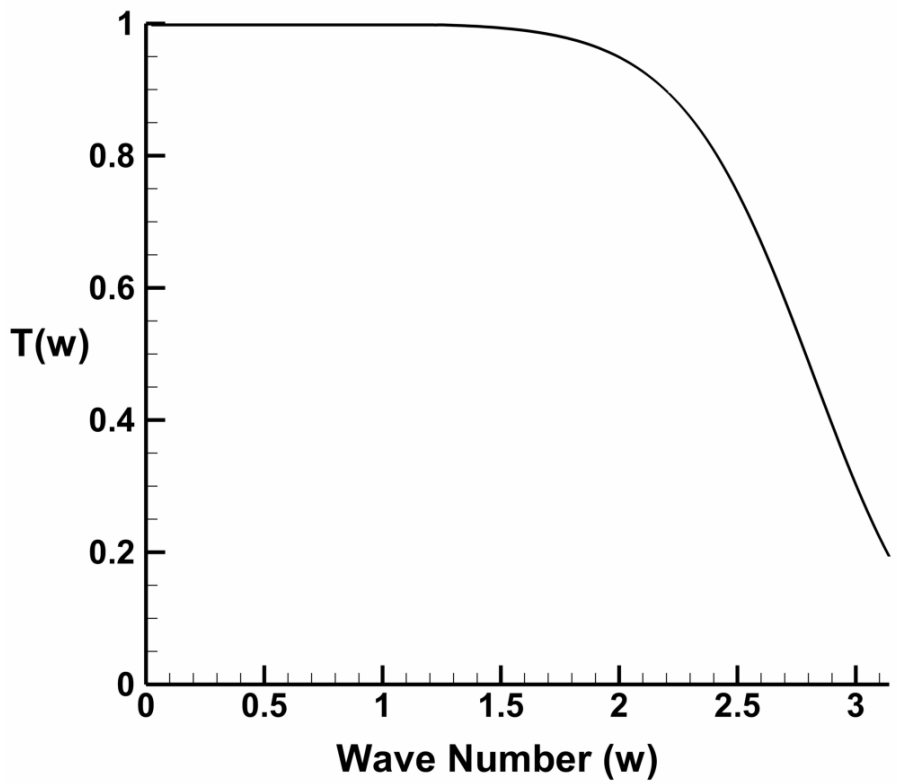

Figure 9. Transfer function versus wave number for filter (equation 19).

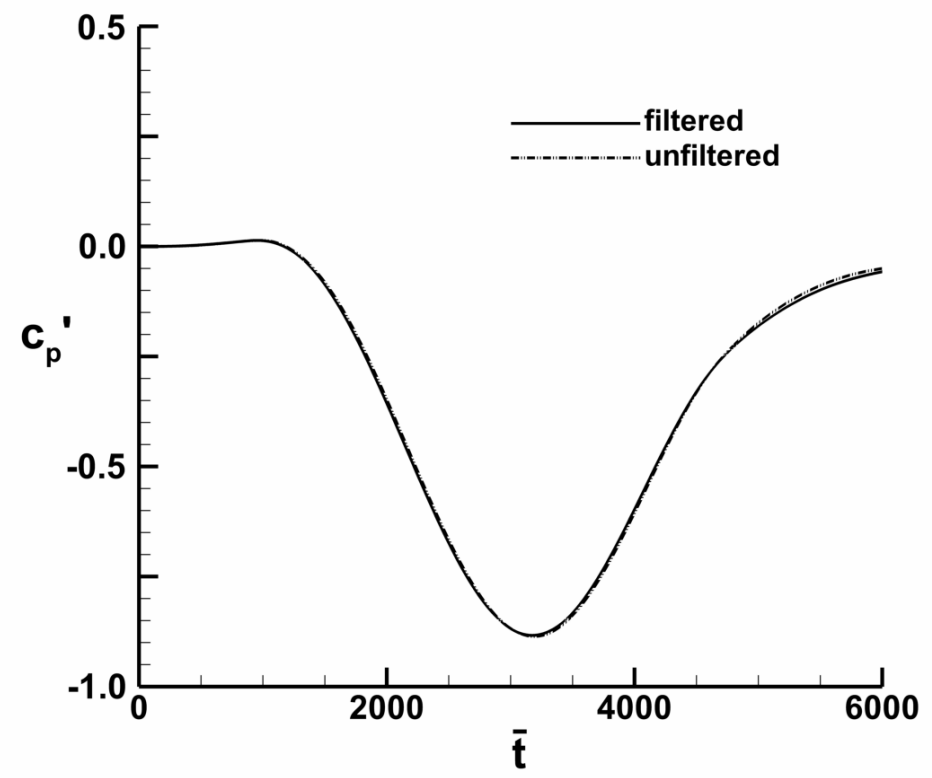

Figure 10. Pressure coefficient time histories due to one-minus-cosine gust, station 6. 


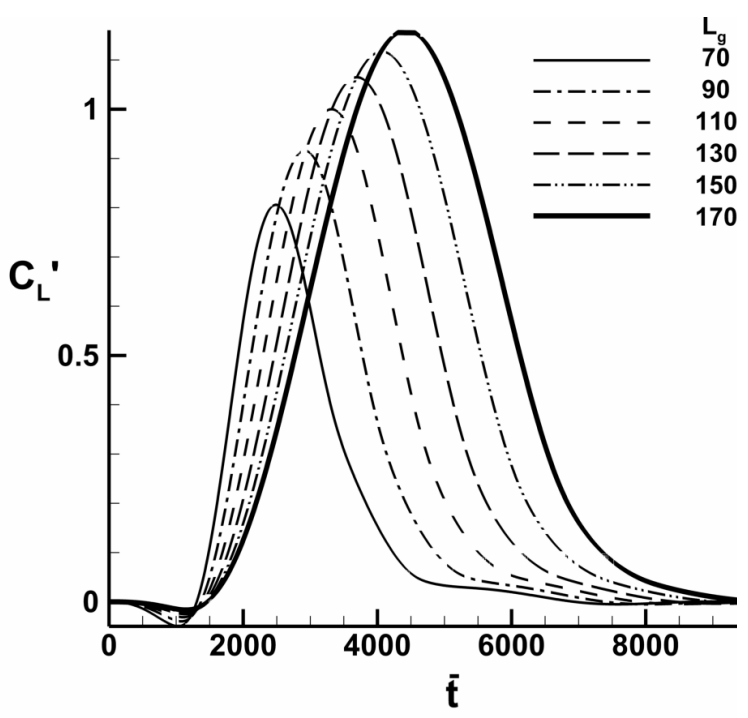

(a) Lift coefficient

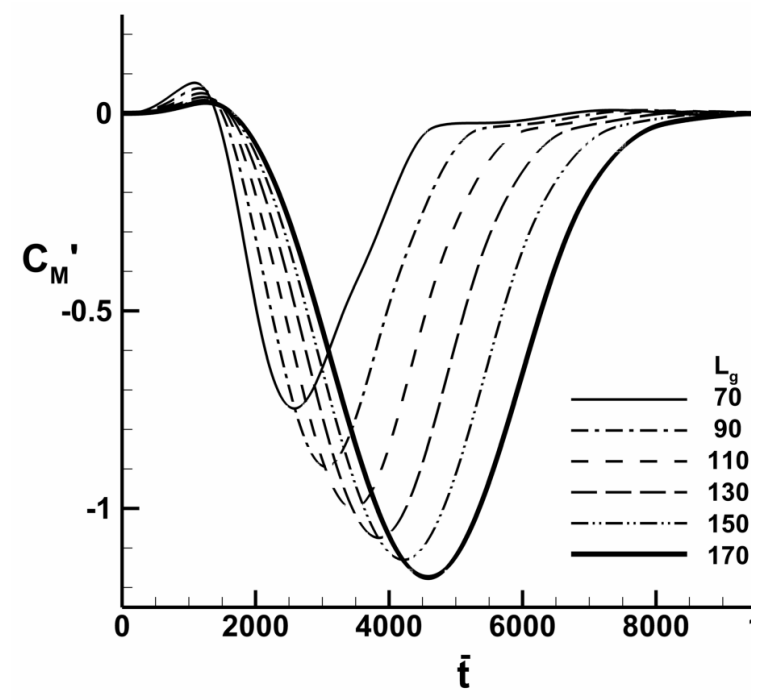

(b) Moment coefficient

Figure 11. Variation of lift and moment coefficient time histories as function of gust length. 